\title{
Accident Detection and Reporting System
}

\author{
Kushal P, Sudarshan K, Akshay Dsouza \\ Computer Science, Srinivas Institute of Technology, Mangalore, Karnataka, India
}

\begin{abstract}
Article Info

Volume 7, Issue 4

Page Number: 271-276

Publication Issue :

July-August-2021

\section{Article History}

Accepted : 15 July 2021

Published : 22 July 2021

A significant rise in the number of road accidents has been observed due to the increasing number of vehicles. Significant research has been conducted on accident for the rescue, particularly on the use of Information and also Communication Technologies for efficient and prompt rescue or saving operations. The majority of such works will provide us the sophisticated solutions that focus on reducing response times. However, such solutions can be very expensive and are not available in all forms of vehicles. Our approach aims to take advantage of the advanced specifications of smartphones to design and develop a low-cost solution for enhanced transportation systems that are deployable in legacy vehicles. In this topic, a customized Android application is developed to collect information regarding speed, attraction, pressure, sound, and location. Speed is one of the factor which is used to improve the identification of the accidents. It arises because of clear differences in environmental conditions or changes (e.g., noise, deceleration rate) that arise in low-speed collisions, versus higher-speed collisions). The information collected is again further processed to detect road incidents. Furthermore, a navigation system is also constructed to report the incident to the nearby hospital. Furthermore, a navigation system is constructed to send and report the accident location and patients details to the nearby hospital! The proposed approach is validated through simulations and comparison with a real data set of road accidents acquired from the Road Safety Open Repository and shows promising results in terms of accuracy.
\end{abstract}

Keywords: World Health Organisation, Safe International Road Travel, Internet of Things, Global Positioning System

\section{INTRODUCTION}

A recent study shows World Health Organisation (WHO) report showed that every year 1.35 million people die and 50 million people get injured. Road accidents are ranked as the eighth leading cause of death, with the Association for Safe International Road Travel (ASIRT) predicting that it may rise to the fifth leading cause of death shortly unless drastic changes occur. ASIRT estimates that between one and two percent of the annual budget of every country is spent on road accidents. Road traffic

Copyright: @ the author(s), publisher and licensee Technoscience Academy. This is an open-access article distributed under the terms of the Creative Commons Attribution Non-Commercial License, which permits unrestricted non-commercial use, distribution, and reproduction in any medium, provided the original work is properly cited 
accidents that will be causing of death by injuring heavily and the tenth main reason of all the deaths globally, now make up a surprisingly significant part of the worldwide burden of ill-health. An approximately estimated 1.2 million people have been killed in street accidents every year! and as many as 50 million more people are injured, occupying 30 percent to 70 percent of orthopaedic beds in developing countries' hospitals. The emergence or the increase of the Internet of Things (IoT) give promise to the development of the intelligent traffic management systems that is Global Positioning System (GPS), is being increasingly used in many of the applications, mainly for vehicle positioning and navigation system. Indeed, so many vehicles which are shipped today will have GPS devices that will sense the position of the vehicle and send this information to cloud servers. Smart cities can provide us the mobility solutions through emerging intelligent transport systems. Many countries are now started implementing real-time intelligent traffic systems which is used to increase safety and reduce pollution. The economic as well as social benefits are also clear; a recent World Bank study finds that "welfare benefits equivalent to 6 percent to 32 percent of the national GDP can be realized from reducing 50 percent of road deaths and injuries for 24 years". One of the effective ways to reduce traffic fatalities is to reduce the response times to collisions. There are much more systems, like eNotify, that can be helpful in detecting and reporting traffic accidents. This may be an effective solution, it is expensive and not all vehicles are equipped with on-board units. The majority of systems being developed are hardware-based and this makes them expensive and not available in every car. In such cases, the sensors are unable to detect and find an accident in that scenario. Smartphone sensors are used to identify accidents and are less likely to be damaged, resulting in fake reporting of an accident. Some existing systems utilize smartphones to detect accidents. However, these systems have been found to have significant fake-positive rates which is known. To overcome these issues, this paper proposes an Accident Detection System that focuses on the accuracy of accident detection and the notification system. The rest of the section is organized as follows: Section 2 presents related research studies on road safety, vehicular communication, and rescue operation after an accident. Section 3 will proposes a modelarchitecture for accident detection as well as reporting. Section 4 tells about the implementation of the proposed system. Section 5 represents the results obtained in the experiments which are performed. Finally, Section 6 will presents the conclusions and future works.

\section{LITERATURE REVIEW}

There are many schemes and techniques in the literature to address road safety, vehicular communication, and rescue operations after an accident. We focus on the most practical solutions and restrict attention to the techniques that are software- and hardware-based systems'. Zhao et al. [1] have proposed a crash notification system that utilizes mobile devices, detecting accidents through accelerometer and GPS data. This system delays in sending the message about an accident that took place. Patel et al. [2] developed an Android application that detects accidents using only accelerometer data. The system automatically sends a pre-recorded voice message to the 108 ambulance services. Khot et al. [3] propose a smartphone-based system that detects an accident using an accelerometer and finds the nearest emergency point to send the location of the accident. Again, this system has the problem of a single point of failure which is leading to a tendency of fake reporting. Zaldivar et al.[4] have developed a smartphone application using an on-board unit. This application will going to enable the driver to speak with his/her 
vehicle. The application detects an accident using airbag triggers and informs the emergency service provider through email or SMS. A drawback of this application is that it requires the smartphone application to be running and we have to download it from respective app stores. Thompson et al.

[5] propose a system that detects accidents using the sensors in a smartphone. The phone uses its $3 \mathrm{G}$ connection or $4 \mathrm{G}$ connection to send the accident information. This system is not fully automated as it sometimes needs a third party to send the emergency information to the responders.

\section{PROPOSED ARCHITECTURE}

To address the current limitations in accident detection systems, we propose an Accident Detection and Reporting System (ADRs). ADRS uses the capabilities of a modern means latest Android smartphone thereby decreasing the overall costing since there is no special hardware requirements. All processing will be performed in the cloud. The system architecture of ADRS is consisting of five different layers, namely the Application layer, the Database layer, the Cloud layer, the Network layer, and the Perception layer. In the proposed architecture, the layer which is responsible for interacting with the sensors of the smartphone is perception layer. The layer which responsible for providing the connectivity between the perception and cloud layers is network layer. The network layer utilizes Wi-Fi or 3G/4G cellular communications for the transmission of data to the cloud layer. The cloud layer holds the algorithm for accident detection and identifies an accident based on threshold analysis. Suppose if an accident is detected, it informs the nearby hospital about the accident. The processing layer transfers the data to the database layer and then finally, the database layer will be responsible for storing the data related to the particular accident, hospital information, driver, and the vehicle information.

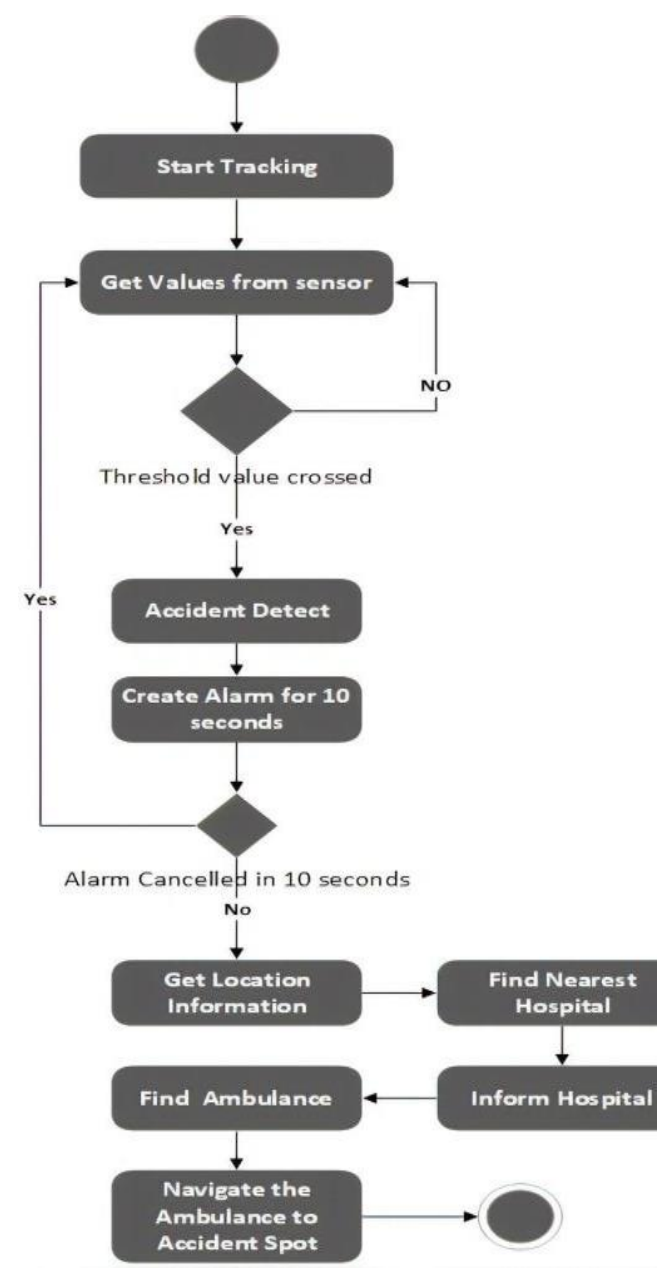

Fig.1 Flowchart of Accident detection and reporting system.

\subsection{WORKING OF PROPOSED SYSTEM}

Figure 3.1 shows the working of the proposed system to give a better understanding of the system. A user or the vehicle driver downloads the application from the Google Play Store using his phone and installs it on their smartphone. After installation, the user will registers the application, providing the required information; after registration, the user can use the application freely. Every time the user commences a journey, he or she activates the tracking process. The smartphone will start reading the sensor data and sends this is the cloud. This information is then 
processed in the cloud to detect any accidents. In the case of an accident, a nearby hospital is informed and presented with details about the accident. The system assumes that each car is connected to the smartphone. Each and every smartphone is consisting of four types of sensors: a pressure sensor; a noise sensor (microphone), an accelerometer, and a speed sensor. For the experimental evaluation, we have used an Android smartphone which is equipped with the aforementioned sensors which will continuously collect raw information from these sensors. The mobile phone continuously sends the data to the cloud which process the data and looks for an accidental condition. The cloud processes data and checks whether an accident has occurred or not. Threshold values are defined and, if the values are collected from the sensors which give rise to a value that exceeds the threshold value, it indicates or shows that an accident has occurred. When these conditions are met, an alarm is produced and sent to the vehicle driver. If the driver is going to cancels the alarm, then the information is not sent to the hospital, to avoid fake reporting. If the driver doesn't respond within the $10 \mathrm{~s}$, the cloud service sends a notification to the nearby hospital. The cloud will contain a database of cars and hospitals. The hospital sends the ambulance to the accident location or area for the rescue operation. The hospitals will also hold a database with the information on the ambulances. The important objective or motive of this architecture is to enhance the accuracy of accident detection.

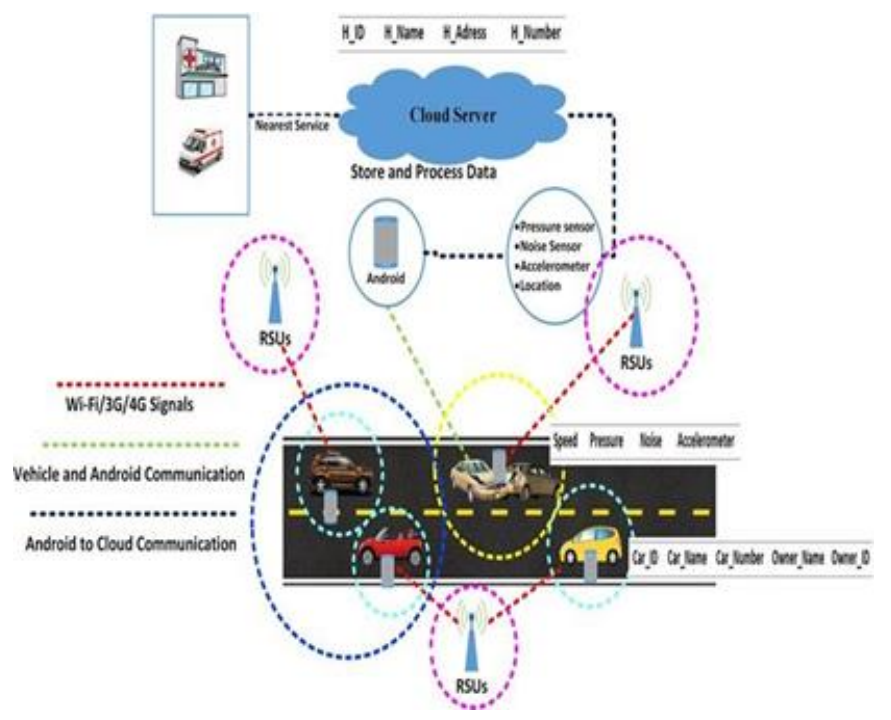

Fig 2 : Working of ADRS

\section{SYSTEM IMPLEMENTATION}

Our proposed system comprises two phases: accident detection and notification phase. In Detection Phase Implementation an Android application has been developed in the Java programming language. The application is developed for an Android operating system with minimum AP! level 17 and target AP!level

A user first registers for system use. Once registered, to use the system, the user will enter their ID and password to log in to the system and they should remember it. Recording as well as transmission of data commences whenever the user will click to start the tracking. The application will continually read the data from the smartphone's sensors and sends the data to the cloud to store or to do some tasks. If an accident is detected, the application generates or creates an alarm for a span of 30s.After an accident is detected, the cloud determines the nearby hospital and informs the hospital about the accident that took place. This will be performed using a web-based application. The application has been developed by using the Vanilla 
JavaScript. This interface will be used by the hospital to establish or create whether there is an emergency or not. Whenever an accident will occur, the website receives or collects the information regarding to the accident. The website will show us the details of accidents such as the location of the accident and driver of the particular vehicle and vehicle information. Google Maps is used to show the position of the accident on a map.

\subsection{Algorithms for accident detection}

Data: Values $\mathrm{f}$ romsmartphoneJsSensors

Result: Status of accident detection; The smartphone performs computations;

if $(\mathrm{G}-$ Force $/ 4+$ Sound $/ 140+$ Pressure/350) $>=(1)$

AND (Speed) $>=(24)$ then

$\mathrm{I} \leftarrow 1$;

else if (G - Force $/ 4+$ sound $/ 140+$ Pressure $/ 350+$ SVP/2.06) $>=(3)$ then

$\mathrm{I} \leftarrow 1$;

else if (G - Force/4 + Sound/140 + Pressure/350) >= (1) AND (ElapsedTime) $<(\mathrm{MP})$ then

$\mathrm{I} \leftarrow 1$;

else

$\mathrm{I} \leftarrow 0$;

end

if $\mathrm{I}=1$ then

START alarm_timer; alarm_timer= 10 seconds; if (alarm_timer) $<=(10$ seconds) then

status= accident_occur; else

status $=$ no_accident;

end

Get location From GPS;

SET location (lat, lang) = current location(lat, lang);

GET car_info from database;

GET emergency_number from database;

MESSAGE = (location, speed, G-force, Owner_Info, emergency_number); CONNECT available WiFi/3G/4 G;

Send MESSAGE to server; else

status $=$ no_accident;

end

\section{CONCLUSION AND FUTURE SCOPE}

In modern cities, the amount of vehicles has been increased drastically in recent years. This increased traffic has increased the number of accidents. While there exist several accident detection systems being brought to market, still a significant number of fatalities arise. e. The lack of availability of effective systems, for affordability and retrofitting capability problems, only exacerbates the isssue. To inform these problems, we have proposed an IoT-based system for accident detection. We have shown that by using a variety of different kind of sensors can be helpful in detecting a road accident more accurately and preicisly. The proposed system immediately finds the location of the accident and calculates the nearby hospital and sends an emergency request for assistance or help to the required hospital department. This system decides based on data received from smartphone sensors, detecting information about the vehicle status. We have performed that our proposed approach lowers the number of fake alarms seen in earlier work. Our system requires Internet connectivity to function. The limitations of our study include that we have to conduct the primary evaluation of the system in a simulated environment which is necessary. Shortly, we will enhance the system by introducing mobile edge computing to reduce latency and enhance security and privacy. Indeed, the system requires full security and privacy analysis, and we intend to address this in future work.

\section{REFERENCES}

[1]. Zhao, Y. Mobile phone location determination and its impact on intelligent transportation systems. IEEE Trans. Intell. Transp. Syst. 2000, 1, 55-64.

[2]. Patel, K. Utilizing the Emergence of Android Smartphones for Public Welfare by Providing Advance Accident Detection and Remedy by 
108 Ambulances. Int. J. Eng. Res. Technol. (IJERT) 2013, 2, 1340-1342.

[3]. Khot, I.; Jadhav, M.; Desai, A.; Bangar, V. Go Safe: Android application for accident detection and notification. Int. Res. J. Eng. Technol. 2018, 5, 4118- 4122.

[4]. Zaldivar, J.; Calafate, C.T.; Cano, J.C.; Manzoni, P. Providing accident detection in vehicular networks throughOBD-II devices and Androidbased smartphones. In Proceedings of the IEEE 36th Conference on Local Computer Networks, Bonn, Germany, 4-7 October 2011; pp. 813819.

[5]. Thompson, C.; White, J.; Dougherty, B.; Albright, A.; Schmidt, D.C. Using smartphones to detect car accidents and provide situational awareness to emergency responders. In Proceedings of the International Conference on Mobile Wireless Middleware, Operating Systems, and Applications, Berlin, Heidelberg, 30 June-2 July 2010; pp. 29-42.

\section{Cite this article as :}

Kushal P, Sudarshan K, Akshay Dsouza, "Accident Detection and Reporting System", International Journal of Scientific Research in Computer Science, Engineering and Information Technology (IJSRCSEIT), ISSN : 2456-3307, Volume 7 Issue 4, pp. 271-276, July-August 2021. Available at doi : https://doi.org/10.32628/CSEIT217478 Journal URL : https://ijsrcseit.com/CSEIT217478 